\title{
New coherent sources for mid infrared spectroscopic applications
}

\author{
F. J. M. Harren \\ Molecular and Laser Physics, Institute for Molecules and \\ Materials, Radboud University, 6525 AJ Nijmegen, the Netherlands
}

F.Harren@science.ru.nl

\begin{abstract}
Summary:
An overview will be given on the new coherent sources that became available and can fulfil the needs for analytical spectroscopy in the mid infrared wavelength region. Recent highly-visible applications will be presented.
\end{abstract}

Keywords: mid-infrared, absorption spectroscopy, trace gas sensing

There is a strong need for new coherent sources in the mid-infrared wavelength region. Mid-infrared spectroscopy is widely used and its application varies from chemical production analysis to calibration of infrared space telescopes. For most of these applications a Fourier Transform Infrared Spectrometers is applied with an infrared lamp. In contrast to a lamp, coherent sources can have long interaction path lengths making them highly sensitive for absorption. Coherent mid-infrared sources were already available directly after the invention of the laser. Consisting of molecular gas discharge lasers, they were able to cover the mid-infrared wavelength region with Watts of laser power. There disadvantage was their spectroscopic tunability, as they only emit at molecular transitions. Diode lasers were only able to be operated at milliWatts power, at liquid nitrogen temperature. With the invention of the Quantum Cascade laser (QCL) and later the Interband Cascade Laser (ICL). This changed and electrically pumped systems became available in which the wavelength emission was not depending on the band-gap of the material, but on the material design. QCLs and ICLs are covering up the mid-infrared wavelength region, with high frequency emission purity and tens of milliWatt (up to Watts) of power in a compact design. Such systems are ideal to operate at a specific infrared wavelength a spectroscopic transition in a sensing system, but is not able to operate as an analytic tool. This changed with the external cavity system designs, in which the coverage of QCLs could be extended over hundreds of wavenumbers.

Alternatively, non-linear generation was also able to cover the mid-infrared wavelength region.
To pump non-linear crystals such as Lithium Niobate, near-infrared technologically very well developed telecom lasers are used. Via Optical Parametric Oscillation, Difference Frequency Generation and their varieties can generate high-power, monochromatic, widely tunable infrared light.

To perform analytical spectroscopy, tunability is needed, next to a wide coverage and a reasonable measuring time period to beat a Fourier Transform Spectrometer. This came available with the development of femtosecond lasers and frequency comb systems. Due to this Nobel Prize winning invention a wide spectral coverage became available with excellent frequency control. Due to dual frequency comb spectroscopy a wide wavelength coverage could be investigated at microsecond timescale. These systems are now full in development for both non-linear generation and direct pumped quantum cascade lasers.

Alternatively, an easier variant generation became available. Due to the high intensity of femto-, pico- and nanosecond pulses in fiber cores non-linear spectral broadening takes place, resulting in fiber-based supercontinuum sources with ultra wide spectral coverage, from the near-infrared to 10 micrometers. Examples will be given of supercontinuum sources and frequency combs that are able to replace partially of completely Fourier Transform Spectrometers outperforming them in interaction path length and time resolution.

Spectroscopic systems are moving towards hyperspectral imaging, combining spectral information and spatial coverage. Recent examples 
are the use of plasmonics combined with mid-infrared spectroscopy determining molecular nanostructures in biological samples. This has a strong potential to transfer the properties of nanophotonics into medical devices for point-of care disease diagnostic. Another highly visible example is the use of microresonators for chemical analysis, combining high-Q resonators with activated surfaces to analyze extremely sensitive, molecular structures with high selectivity making it an ideal tool for analytical chemistry. 